\title{
Changes on the Stokes Shift in Large CdSe Colloidal Quantum Dots by a Ligand Exchange
}

\author{
Sandra Alvarenga, Hamilton Ponce, Ignacio González Oliva, Carlos Rudamas \\ Laboratorio de Espectroscopía Óptica, Escuela de Física \\ Facultad de Ciencias Naturales y Matemáticas, Universidad de El Salvador \\ Final 25 Av. Norte, San Salvador, El Salvador, Central America \\ ar12015@ues.edu.sv; hamilton.ponce@ues.edu.sv; ignacio.gonzalez@ues.edu.sv; carlos.rudamas@ues.edu.sv
}

\begin{abstract}
In this work, we have studied the optical properties of CdSe colloidal quantum dots (CQDs) that undergo a ligand exchange. The QDs were synthesized by the thermal decomposition method and characterized by using optical spectroscopy techniques and Density Functional Theory (DFT) calculations. Trioctylphosphine (TOP) and Oleic acid (OA) ligands were replaced by Formic acid. This ligand exchange seems to cause an increment of the Stokes shift on the nanostructures. As a result a surface underpassivation of the nanostructures appears. This could be related to the generation of non-radiative states that decreases the quantum yield in our samples and a redistribution of the band edge electronic states that explain to some extent our observed results.
\end{abstract}

Keywords: Colloidal Quantum Dots, Ligand Exchange, Optical Properties, Stokes Shift, CdSe, Formic Acid.

\section{Introduction}

Colloidal quantum dots (CQDs) have been studied on the last decades in solid state physics and materials science due to the wide range of applications they have such as bioimaging systems, lasers, LEDs and photovoltaics [1-8]. Some of the CQD properties are the relatively easy synthesis process and the simple tunnability of their emission. The latter, is directly related to QD size, explained by the quantum confinement effect. In these nanostructures, surface interactions play one of the most important roles when it comes to the performance of their optoelectronic properties. During the synthesis process long chain ligands like Trioctylphosphine (TOP) are used. These long chains are capable of giving colloidal stability and assure the formation of the quantum dots. However, these long chains could work against efficient carrier transport [5]. Thus to improve their performance a ligand exchange is required. By performing a ligand exchange, it is possible to obtain results such as enhancement of the efficiency, a broadening of the emission bands, aggregation of the nanostructures and Stokes shift changes [5, 9]. Despite the wide variety of studies related to the characterization of nanostructures, there is still a long way to obtain a better understanding of these effects. In this work, we discuss the effects of ligand exchange on the Stokes shift when Formic acid replaces long chains on the surface of the nanostructures.

\section{Experiment}

Colloidal QDs were synthesized following the thermal decomposition method proposed by Boatman et al. and previously reported in other publications [10-12]. In summary: a Se precursor solution (solution A) is prepared by combining $30 \mathrm{mg}$ of Se, $5 \mathrm{~mL}$ of octadecene and $0.4 \mathrm{~mL}$ of TOP at $200{ }^{\circ} \mathrm{C}$ and cooled at room temperature. A Cd precursor solution (solution B) is later prepared by adding $13 \mathrm{mg}$ of $\mathrm{CdO}, 0.6 \mathrm{~mL}$ of oleic acid (OA) and $10 \mathrm{~mL}$ of octadecene and heated at $225^{\circ} \mathrm{C}$. Then $1 \mathrm{~mL}$ of solution $\mathrm{A}$ is added. Due to the dependence of the resulting size of the QDs with the reaction time, aliquots were taken at different times from the nucleation. They were quenched and stored at room temperature.

The aliquot samples were washed three times in methanol and redispersed in toluene. Formic acid was then added to each sample. Here, the ligand excess was fixed to be around 10 times the concentration of the CdSe atoms in solution. The resulting solution was stored at room temperature. Absorption spectra were measured using an USB4000 UV-VIS spectrograph with a spectral resolution around $1.50 \mathrm{~nm}$, with an entrance slit of $25 \mu \mathrm{m}$ and a Tungsten lamp as light source. PL spectra were measured with an USB4000-FL spectrograph with a spectral resolution around $10 \mathrm{~nm}$, and an entrance slit of $200 \mu \mathrm{m}$ using a LED with $\lambda_{\text {exc }}=395 \mathrm{~nm}$ as excitation source. All of them were manufactured by Ocean Optics. 
To estimate the size of the CQDs we used the semi-empirical model reported by Yu and co-workers [13]. To support this estimation, we have also performed density functional theory (DFT) calculations, as described elsewhere [15].

\section{Results and Discussion}

Figure 1 shows the absorption spectra of several synthesized samples. An absorption band, associated to the band edge absorption, is clearly observed. As stated above, the QD sizes were estimated by using the semi-empirical model reported by $\mathrm{Yu}$ et al., as shown in Figure 2, using the band edge absorption peak energy for each sample. The quantum confined effect is observed here as a red shift of the band edge absorption when the size of the QDs increased. DFT calculations, also shown in Fig. 2, support the interpretation of these results. Despite of the underestimation of the energy of the absorption transition, the behaviour of the absorption transition energy when the QD size increases is similar to the obtained experimental results. The underestimation of the absorption transition energies obtained in DFT calculations is understood and has been explained elsewhere [15]

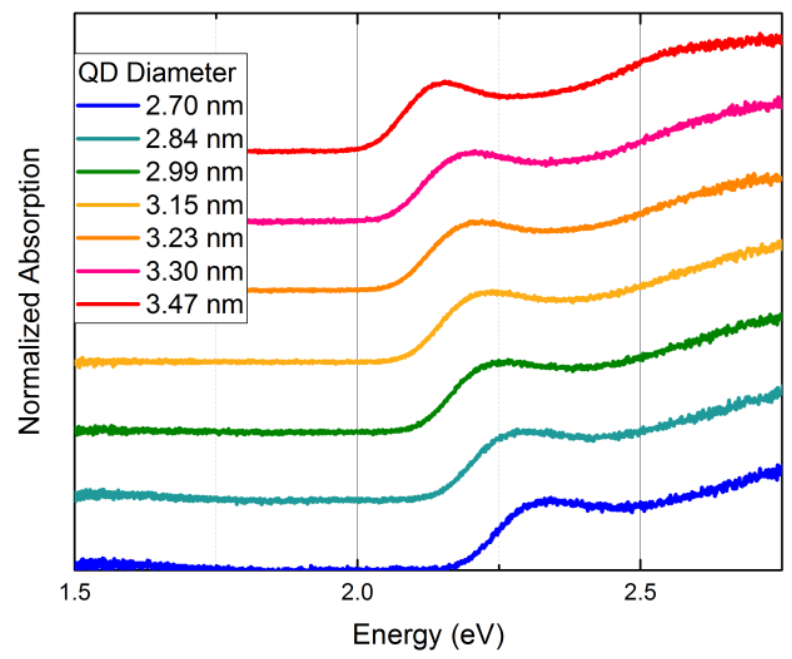

Fig. 1: Normalized absorption spectra. The quantum confinement effect is seen as a red shift in the band edge peak with increasing QD size.

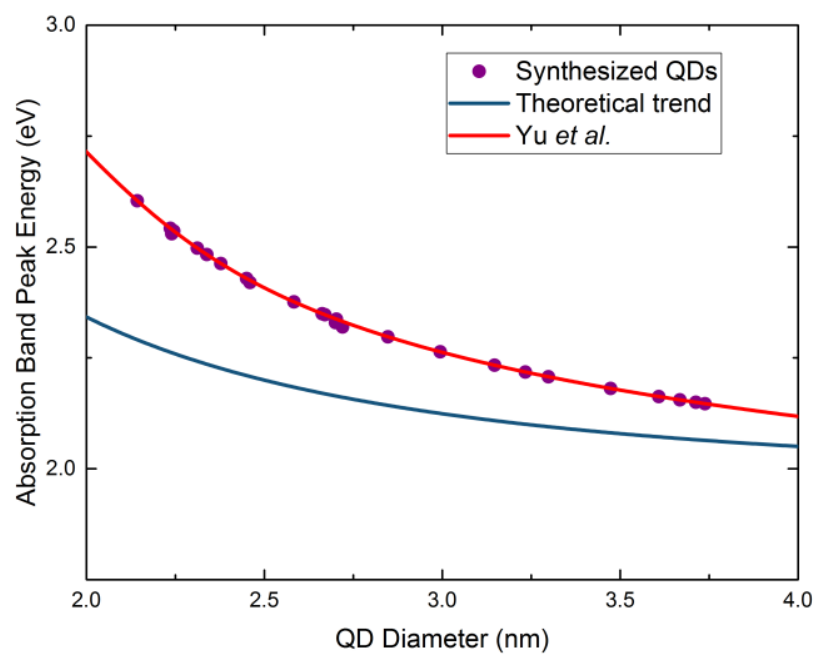

Fig. 2: Theoretical trend and synthesized QD sizes. A similar trend is observed in experimental and theoretical plots. 
Figure 3 shows the PL spectra of the synthesized samples. Two emission bands are clearly observed, especially for small QDs. The band at higher energies is associated to the band edge emission whereas the wide band at lower energies has its origin in the recombination of charge carriers trapped in surface defects [11]. A red shift of the band edge emission is also here observed when the QD size increases. We also observe a decreasing of the surface defect band emission for higher QD sizes. This could be understood considering the decreasing on the surface-to-volume ratio with an increasing of the size of the QDs. The surface-to-volume ratio is inversely proportional to the QDs size, meaning in smaller QDs there is a higher ratio of superficial atoms, relative to the total number of atoms on the structure [16].

Figure 4 shows the absorption and PL spectra for the $3.61 \mathrm{~nm}$ QDs before and after the ligand exchange process was done. A blue shift is clearly observed in the absorption spectrum. The absorption band edge energy peak is shifted from $2.16 \mathrm{eV}$ for TOP/OA capped QDs to $2.19 \mathrm{eV}$ in the case of Formic acid capped QDs. On the other hand, we observe a band edge emission energy peak shift from $2.12 \mathrm{eV}$ to $2.09 \mathrm{eV}$ in the PL spectra. The ligand exchange treatment produced a similar shift in all our absorption and PL spectra for different CdSe nanostructure sizes.

We believe that the increment of the Stokes shift in our samples suggest an underpassivation in our QDs. Accordance to this, we observed (not shown here) an 8-fold decreasing of the quantum yield after the ligand exchange, which could be associated to the generation of non-radiative traps within this underpassivation. In fact, surface modification trough ligand exchange has been reported to cause different effects on the absorption and PL spectra of QDs, where electronic reconfiguration is one of the main causes of these shifts [13, 17-22]. Underpassivation could also cause changes on the band edge states and a decreasing of the oscillator strength in CQDs, as reported by other authors [23]. This is good agreement with our obtained results.

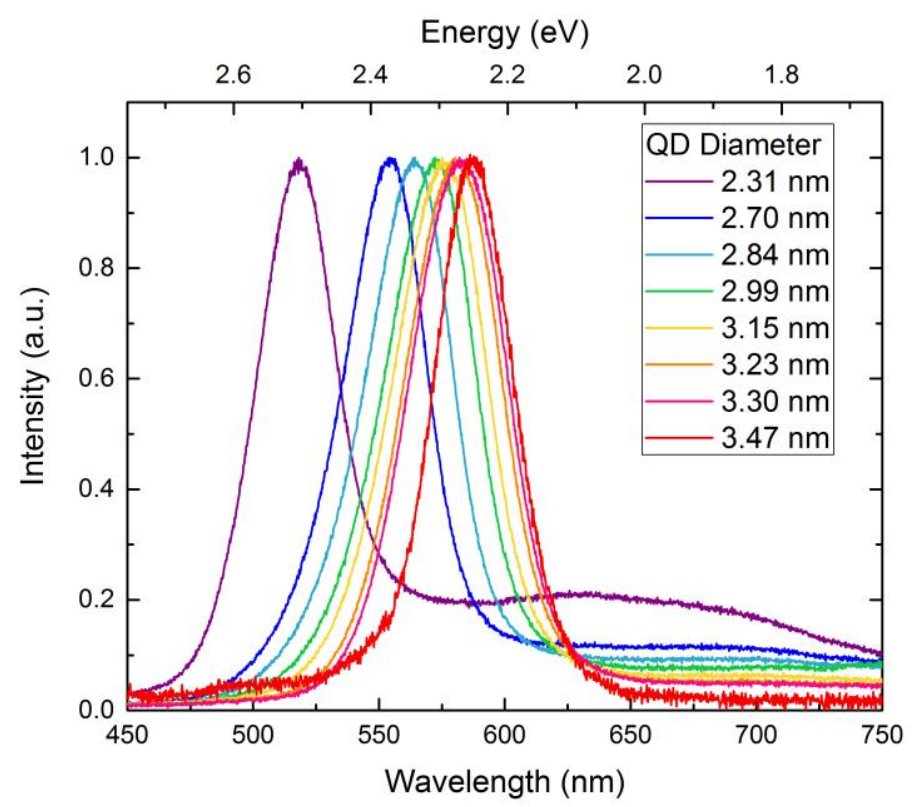

Fig. 3: PL spectra normalized to the band edge maximum for different QD sizes. 


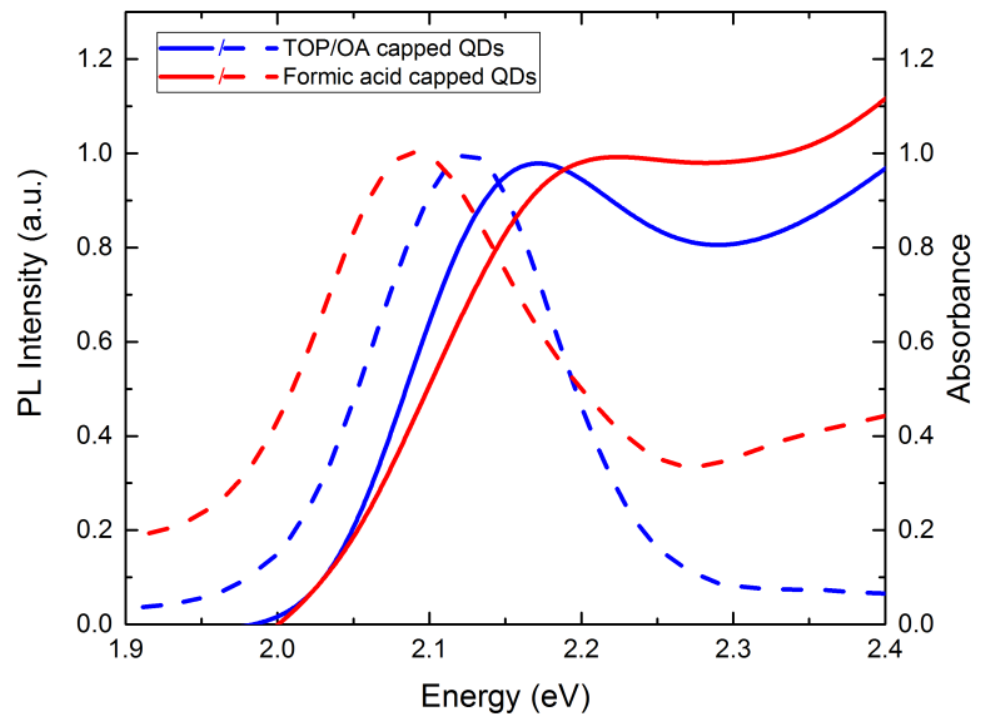

Fig. 4: Normalized absorption (solid lines) and PL spectra normalized to the band edge maximum (dashed lines) for 3.61 nm sized QDs before and after the ligand exchange process.

Formic acid as capping ligand has been used before in ultrasmall CdSe QDs (smaller than $2 \mathrm{~nm}$ in diameter) to study white light emitting QDs [5]. The PL red shift observed in our samples differs from the results presented by Rosenthal et al., but this could be related, to some extent, to the size of the studied nanostructures and/or the difference in primary ligands used on each synthesis process.

\section{Conclusion}

In this work, PL spectra for different QD sizes (QD diameter > $2.31 \mathrm{~nm}$ ) are presented. An increment of the Stokes shift was observed on CdSe CQDs after a ligand exchange process where Formic acid was added as new capping ligand. This could be related to an effect of underpassivation in our QDs after the ligand exchange. The latter generates non-radiative states that compete against an efficient recombination, what we observed as a decreasing of the quantum yield in our samples, and a redistribution of the electronic states that could explain, to some extent, our obtained results.

\section{Acknowledgements}

The authors would like to thank D. Pleitez, W. Abarca, J. Cuadra, and the other members of the research group that contributed to the discussion of these results. This work has been partially supported by Consejo de Investigaciones Científicas (CIC-UES) under the 09.20 project.

\section{References}

[1] F. Dubois, B. Mahler, B. Dubertret, E. Doris and C. Mioskowski, "A versatile strategy for quantum dotlig and exchange," J. Am. Chem. Soc., vol. 129, pp. 482-483, 2007.

[2] K. Das, S. Sanwlani, K. Rawat et al., "Spectroscopic profile of surfactant functionalized CdSe quantum dots and their interaction with globular plasma protein BSA," Colloids and Surfaces A: Physicochem. Eng. Aspects, vol. 506, pp. 495-506, 2016.

[3] V. Klimov et al., "Optical Gain and Stimulated Emission in Nanocrystal Quantum Dots," Science, vol. 290, pp. 314$317,2000$. 
[4] T. Rosson, S. Claiborne, J. McBride et al., "Bright white light emission from ultrasmall cadmium selenide nanocrystals," J. Am. Chem. Soc., vol. 134, pp. 8006-8009, 2012.

[5] J. Tang, K. Kemp, S. Hoogland et al., "Colloidal-quantum-dot photovoltaics using atomic-ligand passivation," Nature Materials, vol. 10, no. 10, pp. 765-771, 2011.

[6] M. Beard, J. Luther, A. Nozik, "Multiple exciton generation in semiconductor quantum dots and electronically coupled quantum dots arrays for applications to third-generation photovoltaic solar cells," Chem. Rev., vol. 110, no. 11, pp. 6873-6890, 2010.

[7] K. M. Kim, J.Hye Jeon, Y. Y. Kim et al., "Effects of ligand exchanged CdSe quantum dot interlayer for inverted organic solar cells," Organic Electronics, vol. 25, pp. 44-49, 2015.

[8] M.Wang, N. Felorzabihi, G. Guerin et al., "Water-Soluble CdSe Quantum Dots Passivated by a Multidentate Diblock Copolymer," Macromolecules, vol. 40, pp. 6377-6384, 2007.

[9] C. Bullen and P. Mulvaney, "The effects of chemisorption on the luminescence of CdSe quantum dots," Langmuir, vol. 22, no. 7, pp. 3007-3013, 2006.

[10] K. Nordell, E. Boatman and G. Lisensky, "A Safer, Easier, Faster Synthesis for CdSe Quantum Dot Nanocrystals," J. Chem. Ed., vol. 82, no. 11, pp. 1697-1699, 2005.

[11] H. Ponce and C. Rudamas, "Optical characterization of surface defects in cadmium selenide (CdSe) colloidal quantum dots," Revista Matèria, vol. 20, no. 3, pp. 987-992, 2013.

[12] M. Pacheco and C. Rudamas, "Fabrication and optical characterization of CdSe quantum dots," in Proceddings of the 62nd Annual Meeting of the Austrian Physical Society, Graz, Austria, pp.159, 2012.

[13] W. Yu, L. Qu, W. Guo et al., "Experimental determination of the extinction coefficient of CdTe, CdSe, and CdS nanocrystals," Chem. Mater, vol. 15, no. 14, pp. 2854-2860, 2003.

[14] M. Grabolle, M. Spieles, V. Lesnyak et al., "Determination of the Fluorescence Quantum Yield of Quantum Dots," Anal. Chem., vol. 81, no. 15, pp. 6285-6294, 2009.

[15] I. González Oliva, S. Alvarenga and C. Rudamas, "A theoretical-experimental comparison of CdSe quantum dot optical properties," in Proceedings of the 2nd World Congress on Recent Advances in Nanotechnology (RAN17), Barcelona, Spain, pp. ICNMS 105-1, 2017.

[16] Y. Yin and A. Alivisatos, "Colloidal nanocrystal synthesis and the organic-inorganic interface," Nature, vol. 437, no. 7059, pp. 664-670, 2005.

[17] D. V. Talapin, A. L. Rogach, A. Kornowski et al., "Highly Luminescent Monodisperse CdSe and CdSe/ZnS Nanocrystals Synthesized in a Hexadecylamine-Trioctylphosphine Oxide- Trioctylphospine Mixture," Nano Letters, vol. 1, no. 4, pp. 207-211, 2001.

[18] X. Luo, P. Liu, N. Truong et al., "Photoluminescence blue-shift of CdSe nanoparticles caused by exchange of surface capping layer," J. Phys. Chem. C, vol. 115, no. 43, pp. 20817-20823, 2011.

[19] R. Koole, P. Schapotschnikow, C. Donega et al., "Time-Dependent Photoluminescence Spectroscopy as a Tool to Measure the Ligand Exchange Kinetics on a Quantum Dot Surface," ACS Nano, vol. 2, no. $\quad$ 8, pp. 1703-1714, 2008.

[20] W. Bae, J. Joo, L. Padilha et al., "Highly effective surface passivation of pbse quantum dots through reaction with molecular chlorine," J. Am. Chem. Soc., vol. 134, 49, pp. 20160-20168, 2012.

[21] M. Schreuder, J. Mcbride, D. Albert et al., "Control of Surface State Emission via Phosphonic Acid Modulation in Ultrasmall CdSe Nanocrystals: The Role of Ligand Electronegativity Control of Surface State Emission via Phosphonic Acid Modulation in Ultrasmall CdSe Nanocrystals: The Role of Ligand Electronegativity," J. Phys. Chem. C., 2019, vol. 113, no. 19, pp. 8169-8176.

[22] C. R. Kagan and C. B. Murray, "Charge transport in strongly coupled quantum dot solids," Nature Nanotechnology, vol. 10, pp. 1013-1026, 2015.

[23] O. Voznyy, F. Fan, A. Ip et al., "Passivation-sensitive exciton finestructure produces excess Stokes shifts in colloidal quantum dots,” H. arXiv:1608.05113. arXiv.org e-Print archive. ～https://arxiv.org/abs/1608.05113. 